\title{
ALGORITHMIC ANALYSIS OF THE SPARRE ANDERSEN MODEL IN DISCRETE TIME
}

\author{
BY
}

\author{
Attahiru Sule Alfa And Steve Drekic
}

\begin{abstract}
In this paper, we show that the delayed Sparre Andersen insurance risk model in discrete time can be analyzed as a doubly infinite Markov chain. We then describe how matrix analytic methods can be used to establish a computational procedure for calculating the probability distributions associated with fundamental ruin-related quantities of interest, such as the time of ruin, the surplus immediately prior to ruin, and the deficit at ruin. Special cases of the model, namely the ordinary and stationary Sparre Andersen models, are considered in several numerical examples.
\end{abstract}

\section{KEYWORDS}

Sparre Andersen model, discrete time, matrix analytic methods, time of ruin, surplus immediately prior to ruin, deficit at ruin.

\section{INTRODUCTION}

In this paper, we consider the delayed Sparre Andersen insurance risk model in discrete time. In particular, we assume that the number of claims process $\left\{N_{t}: t=0,1,2, \ldots\right\}$ is a modified discrete-time renewal process with independent positive interclaim times $\left\{W_{1}, W_{2}, W_{3}, \ldots\right\}$, where $W_{1}$ is the duration from time 0 until the first claim occurs and $W_{i}, i=2,3,4, \ldots$, is the time between the $(i-1)$-th and $i$-th claims. For $i=2,3,4, \ldots$, let $W_{i}$ have probability mass function (pmf) $a_{j}=\operatorname{Pr}\left\{W_{i}=j\right\}, j=1,2,3, \ldots, n_{a}$, and corresponding survival function $A_{j}=\operatorname{Pr}\left\{W_{i}>j\right\}=1-\sum_{k=1}^{j} a_{k}$, where we adopt the usual convention that $\sum_{k=1}^{0} f(k)=0$ for an arbitrary function $f$. We assume in this paper that $n_{a}<\infty$ (i.e., the interclaim time distribution of $W_{i}, i=2,3,4, \ldots$, has finite support).

In the ordinary Sparre Andersen model, it is assumed that a claim has occurred at time 0 , so that $W_{1}$ has the same distribution as the ordinary interclaim times $\left\{W_{2}, W_{3}, W_{4}, \ldots\right\}$. If $W_{1}$ is not a "full" interclaim time, however, it is well known from standard renewal theory (e.g., see Karlin and Taylor (1975, pp. 192-193)) that, asymptotically in time, the limiting distribution of this forward recurrence 
time is defined by the pmf $\tilde{a}_{j}=A_{j-1} / \sum_{k=1}^{n_{a}} A_{k-1}, j=1,2,3, \ldots, n_{a}$. As a result, in the stationary Sparre Andersen model, $W_{1}$ has pmf $\tilde{a}_{j}$ rather than $a_{j}$. Since the motivation for the use of $\tilde{a}_{j}$ is asymptotic in nature, it is not evident how appropriate this assumption is for finite time. To accommodate other possible alternatives, we assume more generally that $W_{1}$ has $\operatorname{pmf} r_{j}=\operatorname{Pr}\left\{W_{1}=j\right\}, j=1$, $2,3, \ldots, n_{r}$, where $n_{r}<\infty$. By appropriate choice of $r_{j}$, it is obvious that both the ordinary and stationary Sparre Andersen models are special cases of this more general risk model.

We further assume that the individual claim amounts $\left\{Y_{1}, Y_{2}, Y_{3}, \ldots\right\}$ form an independent and identically distributed (iid) sequence of positive random variables with common pmf $\alpha_{j}, j=1,2,3, \ldots, m_{\alpha}$, and corresponding survival function $\Lambda_{j}=1-\sum_{k=1}^{j} \alpha_{k}$. We remark that unlike the interclaim time distributions defined above, the claim amount distribution can be of finite or infinite support (i.e., $m_{\alpha} \leq \infty$ ). Premiums are collected at the rate of $c \in \mathbb{Z}^{+}$per unit time. Beginning with an initial reserve of $u \in\{0,1,2, \ldots\}$, the insurer's surplus at time $t$ is given by $U_{t}=u+c t-\sum_{i=1}^{N_{t}} Y_{i}, t=0,1,2, \ldots$ At any given time point, we adopt the usual convention that premiums are collected first before any claims are paid. Let $T=\min \left\{t \in \mathbb{Z}^{+}: U_{t}<0\right\}$ be defined as the time of ruin with $T=\infty$ if $U_{t} \geq 0 \forall t \in \mathbb{Z}^{+}$. If ruin does occur, we also define $\left|U_{T}\right|$ as the deficit at ruin and $U_{T-}=U_{T-1}+c$ as the surplus immediately prior to ruin. Clearly, $T=\infty$ if $m_{\alpha} \leq c$. If $m_{\alpha}>c$, however, then $\left|U_{T}\right| \in\left\{1,2,3, \ldots, m_{\alpha}-c\right\}$ and $U_{T-} \in$ $\left\{c, c+1, c+2, \ldots, m_{\alpha}-1\right\}$.

It is of considerable interest to risk practitioners to be able to calculate the joint probability distribution (as well as associated marginal distributions) of $T, U_{T-}$, and $\left|U_{T}\right|$. Surprisingly, however, there appear to be few results in the literature for computing the joint probability distribution of these fundamental ruin-related quantities of interest in the delayed Sparre Andersen model described above. Most of the results in the literature tend to be specialized and mainly concern the computation of ruin probabilities over finite- and infinite-time horizons for the well-known compound binomial model - a discrete analogue of the classical compound Poisson risk model in which interclaim times are geometrically distributed and the ordinary and stationary variants are identical (e.g., see Gerber (1988), Michel (1989), Shiu (1989), Dickson and Waters (1991), Willmot (1993), Dickson (1994), De Vylder and Marceau (1996), Cheng et al. (2000), Cardoso and Egidio dos Reis (2002), and Li and Garrido (2002)). For the compound binomial model, however, Dickson et al. (1995) developed recursive numerical procedures for calculating the joint and marginal probability distributions of the surplus immediately prior to ruin and the deficit at ruin. While recent papers by Pavlova and Willmot (2004), Li (2005a, 2005b), and Cossette et al. (2006) have analyzed variants of the discrete-time Sparre Andersen model described above, the emphasis in these papers has been primarily theoretical, focussing on bounds on ruin probabilities as well as distributional properties and mathematical connections to other related renewal risk models of interest via the methodology of the Gerber-Shiu discounted penalty function (e.g., see Gerber and Shiu (1998)). 
The thrust of this paper is more computational in flavour. To begin with, we will show how the delayed Sparre Andersen model described above can be set up as a doubly infinite Markov chain with finite blocks. Using this set-up, we will then show how matrix analytic methods can be employed as a means of computing the bivariate joint probabilities

$$
\omega_{n, i}(u)=\operatorname{Pr}\left\{T=n, U_{T-}=i \mid U_{0}=u\right\}, n \in \mathbb{Z}^{+} ; i=c, c+1, c+2, \ldots, m_{\alpha}-1
$$

and

$$
\phi_{n, j}(u)=\operatorname{Pr}\left\{T=n,\left|U_{T}\right|=j \mid U_{0}=u\right\}, n \in \mathbb{Z}^{+} ; j=1,2,3, \ldots, m_{\alpha}-c,
$$

as well as the trivariate joint probability

$$
\begin{aligned}
\psi_{n, i, j}(u)=\operatorname{Pr}\left\{T=n, U_{T-}=\right. & \left.i,\left|U_{T}\right|=j \mid U_{0}=u\right\}, n \in \mathbb{Z}^{+} ; \\
& i=c, c+1, c+2, \ldots, m_{\alpha}-1 ; \\
& j=1,2,3, \ldots, m_{\alpha}-i .
\end{aligned}
$$

Finally, we will demonstrate how these discrete-time probabilities can be employed to approximate analogous quantities in the continuous-time version of this model, where considerably more attention has been devoted in recent years to characterizing the probability distributions of the time of ruin, the surplus immediately prior to ruin, and the deficit at ruin.

\section{FORMULATION OF THE MODEL}

We begin by considering the interclaim time distribution defined by the pmf $a_{j}$. Letting $W$ denote an arbitrary $W_{i}, i=2,3,4, \ldots$, we introduce $\tau_{j}=\operatorname{Pr}\{W>j \mid$ $W>j-1\}=A_{j} / A_{j-1}, j=1,2,3, \ldots, n_{a}$. We immediately note that $\tau_{1}=A_{1}$ and $\tau_{n_{a}}=0$. If we now define the $n_{a} \times n_{a}$ probability transition matrix

$$
S=\left(\begin{array}{ccccc}
0 & \tau_{1} & 0 & \cdots & 0 \\
0 & 0 & \tau_{1} & \ddots & 0 \\
\vdots & \vdots & \ddots & \ddots & \vdots \\
0 & 0 & \cdots & 0 & \tau_{n_{a}-1} \\
0 & 0 & \cdots & 0 & 0
\end{array}\right),
$$

and the $1 \times n_{a}$ row vector $\boldsymbol{e}_{1}=(1,0,0, \ldots, 0)$, Alfa and Neuts (1995) have shown that the random variable $W$ can be modelled as a discrete phase-type random variable with representation $\left(\boldsymbol{e}_{1}, S\right)$ of order $n_{a}$. Alfa and Neuts (1995) refer to this as the elapsed time representation of the discrete random variable $W$. We remark that one may interpret $\left\{1,2,3, \ldots, n_{a}\right\}$ as the transient states of the underlying Markov chain having probability transition matrix $S$ among these states, whereas state $n_{a}+1$ is the absorbing state (i.e., the state which marks the 
end of an interclaim time and the subsequent occurrence of a claim). If we then define the $n_{a} \times 1$ column vector of absorption probabilities

$$
\boldsymbol{s}=\left(\begin{array}{c}
1-\tau_{1} \\
1-\tau_{2} \\
\vdots \\
1-\tau_{n_{a}-1} \\
1
\end{array}\right),
$$

it can be shown that

$$
a_{j}=\boldsymbol{e}_{1} S^{j-1} \boldsymbol{s}, j=1,2,3, \ldots, n_{a} .
$$

Further details concerning this result can be found in Alfa and Neuts (1995) as well as in Alfa (2004).

Consider now the delayed Sparre Andersen model described in Section 1. Suppose we fix the value of $W_{1}$ to be $k$, where $k \in\left\{1,2,3, \ldots, n_{r}\right\}$. Assuming that $W_{1}=k$, define $L_{t}$ as the "elapsed time" (in the sense described above) at time $t, t=k, k+1, k+2, \ldots$, since the occurrence of the last claim. For $t=k, k+1$, $k+2, \ldots$, consider the bivariate stochastic process $\left(U_{t}, L_{t}\right)$ which possesses the following Markovian relationship, namely

$$
\left(U_{t+1}, L_{t+1}\right)= \begin{cases}\left(U_{t}+c, L_{t}+1\right) & \text { if there is no claim at time } t+1 \\ \left(U_{t}+c-Y, 1\right) & \text { if there is a claim } Y \text { at time } t+1 .\end{cases}
$$

Figure 1 depicts a simple transition scheme of the process assuming that $u=6$, $c=1, k=3$, and $U_{3}=5$. An important observation is that $L_{k}=1$, since we are assuming that a claim has occurred at time $k$. Let $\Delta=\left\{\left(U_{t}, L_{t}\right): U_{t} \in \mathbb{Z} ; L_{t}=\right.$ $\left.1,2,3, \ldots, n_{a}\right\}$ denote the state space for this Markov chain. We refer to the $U_{t}$ component as the level of the process and the $L_{t}$ component as the phase of the process. Since the delayed process reverts to the ordinary process (having interclaim time distribution defined by the $\mathrm{pmf} a_{j}$ ) upon occurrence of the first claim, the probability transition matrix $P$ associated with this Markov chain for $t=k, k+1, k+2, \ldots$ is given as

$$
\left.P=\begin{array}{r|ccccccccccccc} 
& \ldots & -3 & -2 & -1 & 0 & 1 & 2 & \ldots & c & c+1 & c+2 & c+3 & \ldots \\
-3 & \ddots & \ddots & \ddots & \ddots & & & & & & & & & \\
-2 & \ddots & B_{c} & B_{c-1} & B_{c-2} & B_{c-3} & & & & & & & & \\
-1 & \ddots & B_{c+1} & B_{c} & B_{c-1} & B_{c-2} & B_{c-3} & & & & & & & \\
0 & \ddots & B_{c+2} & B_{c+1} & B_{c} & B_{c-1} & B_{c-2} & B_{c-3} & & & & & & \\
1 & \ddots & B_{c+3} & B_{c+2} & B_{c+1} & B_{c} & B_{c-1} & B_{c-2} & \ldots & B_{0} & & & & \\
2 & \ddots & B_{c+4} & B_{c+3} & B_{c+2} & B_{c+1} & B_{c} & B_{c-1} & \ldots & B_{1} & B_{0} & & & \\
3 & \ddots & B_{c+5} & B_{c+4} & B_{c+3} & B_{c+2} & B_{c+1} & B_{c} & \ldots & B_{2} & B_{1} & B_{0} & & \\
\vdots & \ddots & B_{c+6} & B_{c+5} & B_{c+4} & B_{c+3} & B_{c+2} & B_{c+1} & \ldots & B_{3} & B_{2} & B_{1} & B_{0} & \\
& \ddots & \ddots & \ddots & \ddots & \ddots & \ddots & \ddots & \ddots & \ddots & \ddots & \ddots & \ddots & \ddots
\end{array}\right),
$$


ALGORITHMIC ANALYSIS OF THE SPARE ANDERSEN MODEL

297

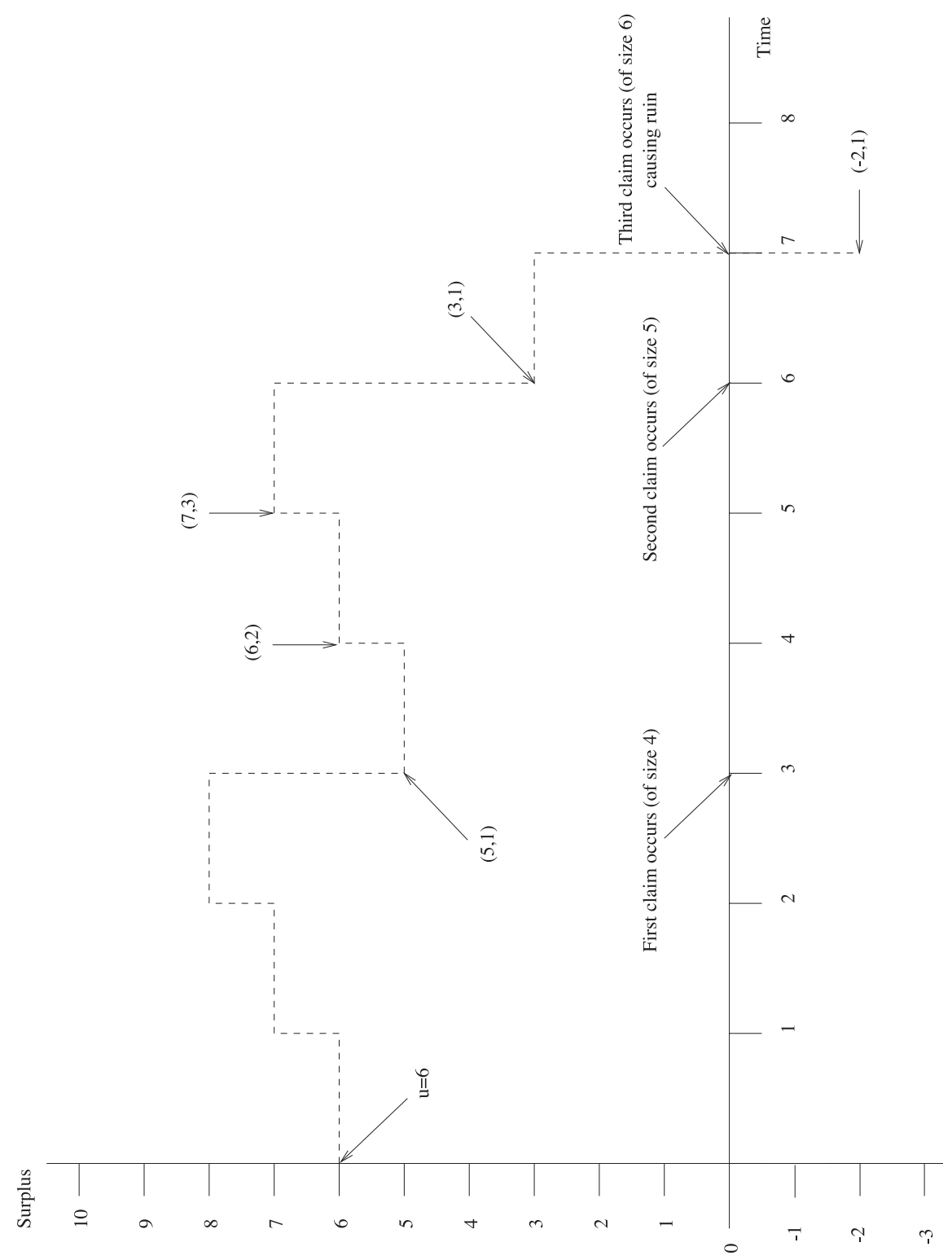

https://doi.org/10.2143/AST.37.2.2024068 Published online by Cambridge University Press 
where

$$
B_{i}= \begin{cases}S & \text { if } i=0, \\ \left(\boldsymbol{s e}_{1}\right) \alpha_{i} & \text { if } i=1,2,3, \ldots\end{cases}
$$

For $c=1$, we note that this is a doubly infinite, right skip-free Markov chain, with finite blocks of size $n_{a}$. Moreover, since $\alpha_{i}=0 \forall i>m_{\alpha}, B_{i}$ becomes an $n_{a} \times n_{a}$ matrix of zeros if $i>m_{\alpha}$.

We now partition the state space $\Delta$ into two state spaces, namely

$$
\Delta_{1}=\left\{(i, j): i=0,1,2, \ldots ; j=1,2, \ldots, n_{a}\right\}
$$

and

$$
\Delta_{2}=\left\{(i, j): i=-1,-2,-3, \ldots ; j=1,2, \ldots, n_{a}\right\} .
$$

Note that $\Delta_{1}$ comprises the "non-ruined" states of the system whereas $\Delta_{2}$ comprises the "ruined" states of the system, so that $\Delta=\Delta_{1} \cup \Delta_{2}$. Moreover, we define two matrices $C$ and $D$ such that $C: \Delta_{1} \rightarrow \Delta_{1}$ and $D: \Delta_{1} \rightarrow \Delta_{2}$. Therefore, the matrix $C$ has block elements $C_{v, w}$ containing transition probabilities which map $U_{t}=v \in\{0,1,2, \ldots\}$ into $U_{t+1}=w \in\{0,1,2, \ldots\}$. Clearly, the non-zero block elements are given as $C_{v, w}=B_{v-w+c}, w=0,1, \ldots, v+c$. In a similar fashion, the matrix $D$ has block elements $D_{v, w}=B_{v-w+c}$, which contain the transition probabilities mapping $U_{t}=v \in\{0,1,2, \ldots\}$ into $U_{t+1}=w \in\{-1,-2,-3, \ldots\}$. As a result, the two matrices $C$ and $D$ look as follows:

$$
C=\begin{gathered}
0 \\
0 \\
1 \\
2 \\
\vdots
\end{gathered}\left(\begin{array}{cccccccc}
B_{c} & B_{c-1} & B_{c-2} & \ldots & B_{0} & & & \\
B_{c+1} & B_{c} & B_{c-1} & \ldots & B_{1} & B_{0} & & \\
B_{c+2} & B_{c+1} & B_{c} & \ldots & B_{2} & B_{1} & B_{0} & \\
\vdots & \ddots & \ddots & \ddots & \ddots & \ddots & \ddots & \ddots
\end{array}\right)
$$

and

$$
D=\begin{gathered}
-1 \\
0 \\
1 \\
2 \\
\vdots
\end{gathered}\left(\begin{array}{cccc}
B_{c+1} & B_{c+2} & B_{c+3} & \cdots \\
B_{c+2} & B_{c+3} & B_{c+4} & \cdots \\
B_{c+3} & B_{c+4} & B_{c+5} & \cdots \\
\vdots & \vdots & \vdots &
\end{array}\right)
$$

We remark that the matrix $C$ is the lower right quadrant of $P$ and the matrix $D$ is the lower left quadrant of $P$ horizontally reversed.

Assuming that $W_{1}=k$, it readily follows that

$$
\operatorname{Pr}\left\{U_{k}=v \mid W_{1}=k, U_{0}=u\right\}=\alpha_{u+c k-v}, v=u+c k-1, u+c k-2, u+c k-3, \ldots
$$


Therefore, the initial (i.e., starting at time $k$ ) probability row vector corresponding to the states in $\Delta_{1}$ is given by

$$
\boldsymbol{b}^{(k)}=\left(\alpha_{u+c k} \boldsymbol{e}_{1}, \alpha_{u+c k-1} \boldsymbol{e}_{1}, \alpha_{u+c k-2} \boldsymbol{e}_{1}, \ldots, \alpha_{2} \boldsymbol{e}_{1}, \alpha_{1} \boldsymbol{e}_{1}, \mathbf{0}, \mathbf{0}, \ldots\right),
$$

where $\mathbf{0}$ denotes the $1 \times n_{a}$ row vector of zeros. Note that the $i$-th level of $\boldsymbol{b}^{(k)}$ is given by the $1 \times n_{a}$ row vector $\alpha_{u+c k-i} \boldsymbol{e}_{1}$ for each $i \in \Omega_{k}=\{0,1,2, \ldots, u+c k-1\}$. Moreover, $\boldsymbol{b}^{(k)}$ contains zeros from level $u+c k$ onward.

We now define two additional row vectors, namely

$$
\boldsymbol{g}_{n}^{(k)}=\left(\boldsymbol{g}_{n, 0}^{(k)}, \boldsymbol{g}_{n, 1}^{(k)}, \boldsymbol{g}_{n, 2}^{(k)}, \ldots\right)=\boldsymbol{b}^{(k)} C^{n}, n=0,1,2, \ldots
$$

and

$$
\boldsymbol{h}_{n}^{(k)}=\left(\boldsymbol{h}_{n,-1}^{(k)}, \boldsymbol{h}_{n,-2}^{(k)}, \boldsymbol{h}_{n,-3}^{(k)}, \ldots\right)=\boldsymbol{g}_{n-1}^{(k)} D=\boldsymbol{b}^{(k)} C^{n-1} D, n=1,2,3, \ldots
$$

Note that $\boldsymbol{g}_{n}^{(k)}$ contains the probabilities of being in the various "non-ruined" states at time $k+n$ without having visited a "ruined" state during the previous $n-1$ transitions, given that $U_{k} \in \Omega_{k}$ according to the probability vector $\boldsymbol{b}^{(k)}$. In a similar fashion, $\boldsymbol{h}_{n}^{(k)}$ contains the probabilities of being in the various "ruined" states for the first time at time $k+n$, given that $U_{k} \in \Omega_{k}$ according to the probability vector $\boldsymbol{b}^{(k)}$. Since ruin can only occur at claim instants (thereby resulting in the phase component being reset to 1 with probability 1 at such instants), it immediately follows that the structure of the $1 \times n_{a}$ row vector $\boldsymbol{h}_{n,-j}^{(k)}$ is simply given by $\boldsymbol{h}_{n,-j}^{(k)}=\left(\phi_{n, j}^{(k)}(u), 0,0, \ldots, 0\right)$, where $\phi_{n, j}^{(k)}(u)=\operatorname{Pr}\{T=k+n$, $\left.\left|U_{T}\right|=j \mid U_{k} \in \Omega_{k}\right\}$. Hence, we simply obtain

$$
\phi_{n, j}^{(k)}(u)=\boldsymbol{h}_{n,-j}^{(k)} \boldsymbol{e}_{1}^{\prime},
$$

where $\boldsymbol{e}_{1}^{\prime}$ denotes the transpose of $\boldsymbol{e}_{1}$.

We can also apply similar probabilistic reasoning to obtain a representation for $\psi_{n, i, j}^{(k)}(u)=\operatorname{Pr}\left\{T=k+n, U_{T-}=i,\left|U_{T}\right|=j \mid U_{k} \in \Omega_{k}\right\}$. In order for ruin to occur at time $k+n$ with a surplus prior to ruin equal to $i$, we observe that: (i) none of the previous $n-1$ transitions must have included a visit to any state in $\Delta_{2}$, and (ii) the surplus at time $k+n-1$ must be equal to $i-c$. The quantity corresponding to points (i) and (ii) is the $1 \times n_{a}$ row vector $\boldsymbol{g}_{n-1, i-c}^{(k)}$. At the next time unit (i.e., time $k+n$ ), a claim must necessarily occur but not before a premium of $c$ is first collected, thereby raising the surplus level to $i$. Since $\boldsymbol{s}$ contains the absorption (to claim occurrence) probabilities from the $n_{a}$ possible phase states, and the claim causing ruin must be of size $i+j$ in order to ensure that the deficit at ruin is equal to $j$, it immediately follows that

$$
\psi_{n, i, j}^{(k)}(u)=\left(\boldsymbol{g}_{n-1, i-c}^{(k)} \boldsymbol{s}\right) \alpha_{i+j} .
$$

Our analysis up to this point has assumed that $W_{1}=k$, where $k \in\left\{1,2,3, \ldots, n_{r}\right\}$. Therefore, conditioning on the value of $W_{1}$ yields, by the Law of Total Probability, 


$$
\phi_{n, j}(u)=\sum_{k=1}^{n_{r}} r_{k} \phi_{n-k, j}^{(k)}(u), n=n_{r}+1, n_{r}+2, n_{r}+3, \ldots
$$

and

$$
\psi_{n, i, j}(u)=\sum_{k=1}^{n_{r}} r_{k} \psi_{n-k, i, j}^{(k)}(u), n=n_{r}+1, n_{r}+2, n_{r}+3, \ldots
$$

However, in order for $T=n$ where $n=1,2,3, \ldots, n_{r}$, it must be that either: (i) time $n$ is the time of the first claim which happens to cause ruin, or (ii) the first claim occurs at some time $k \in\{1,2,3, \ldots, n-1\}$ which does not cause ruin, but ruin subsequently occurs $n-k$ time units later. Mathematically, this translates to

$$
\phi_{n, j}(u)=\sum_{k=1}^{n-1} r_{k} \phi_{n-k, j}^{(k)}(u)+r_{n} \alpha_{u+c n+j}, \quad n=1,2,3, \ldots, n_{r}
$$

and

$$
\psi_{n, i, j}(u)=\sum_{k=1}^{n-1} r_{k} \psi_{n-k, i, j}^{(k)}(u)+\delta_{i, u+c n} r_{n} \alpha_{u+c n+j}, \quad n=1,2,3, \ldots, n_{r},
$$

where $\delta_{i, u+c n}$ denotes the Kronecker delta of $i$ and $u+c n$. Since $r_{n}=0 \forall n>n_{r}$, equations (2.11) and (2.13) (as well as equations (2.12) and (2.14)) can be combined to give

$$
\phi_{n, j}(u)=\sum_{k=1}^{\min \left\{n-1, n_{n}\right\}} r_{k} \phi_{n-k, j}^{(k)}(u)+r_{n} \alpha_{u+c n+j}, \quad n \in \mathbb{Z}^{+}
$$

and

$$
\psi_{n, i, j}(u)=\sum_{k=1}^{\min \left\{n-1, n_{r}\right\}} r_{k} \psi_{n-k, i, j}^{(k)}(u)+\delta_{i, u+c n} r_{n} \alpha_{u+c n+j}, \quad n \in \mathbb{Z}^{+} .
$$

For fixed $j \in\left\{1,2,3, \ldots, m_{\alpha}-c\right\}$, we simply note that summing (2.16) from $i=c$ to $m_{\alpha}-j$ yields (2.15), as required. Similarly, for fixed $i \in\{c, c+1, c+2, \ldots$, $\left.m_{\alpha}-1\right\}$, summing (2.16) from $j=1$ to $m_{\alpha}-i$ yields

$$
\begin{aligned}
\omega_{n, i}(u)= & \sum_{k=1}^{\min \left\{n-1, n_{r}\right\}} r_{k} \sum_{j=1}^{m_{\alpha}-i} \psi_{n-k, i, j}^{(k)}(u)+\delta_{i, u+c n} r_{n} \sum_{j=1}^{m_{\alpha}-i} \alpha_{u+c n+j} \\
= & \Lambda_{i} \sum_{k=1}^{\min \left\{n-1, n_{r}\right\}} r_{k}\left(\boldsymbol{g}_{n-k-1, i-c}^{(k)} \boldsymbol{s}\right)+\delta_{i, u+c n} r_{n}\left(\Lambda_{u+c n}-\Lambda_{u+c n+m_{\alpha}-i}\right), \\
& n \in \mathbb{Z}^{+},
\end{aligned}
$$

where (2.10) was used to establish the last equality. Clearly, $\Lambda_{u+c n+m_{\alpha}-i}=0$ in (2.17) if $m_{\alpha}=\infty$. 


\section{Computational procedure}

In order to calculate the probability distributions of the ruin-related quantities of interest, it is clear from (2.9) and (2.10) that we need to be able to compute the row vectors $\boldsymbol{g}_{n}^{(k)}$ and $\boldsymbol{h}_{n}^{(k)}$, quantities which essentially characterize a matrixbased means of sample path enumeration via (2.7) and (2.8) respectively. At first glance, however, this is not entirely straightforward since $C$ and $D$ are both infinite-dimensional matrices defined by (2.4) and (2.5) respectively. In what follows, we capitalize on the structure of these matrices to develop an efficient and stable computational procedure which ultimately enables one to calculate quantities (2.15), (2.16), and (2.17).

We begin by observing that in the computation of $\boldsymbol{g}_{1}^{(k)}$ given by (2.7), we pre-multiply $C$ by the row vector $\boldsymbol{b}^{(k)}$ given by (2.6). Since $\boldsymbol{b}^{(k)}$ contains zeros from level $u+c k$ onward, we obtain that $g_{1}^{(k)}$ is of the form

$$
\boldsymbol{g}_{1}^{(k)}=\left(\boldsymbol{g}_{1,0}^{(k)}, \boldsymbol{g}_{1,1}^{(k)}, \boldsymbol{g}_{1,2}^{(k)}, \ldots, \boldsymbol{g}_{1, u+c(k+1)-2}^{(k)}, \boldsymbol{g}_{1, u+c(k+1)-1}^{(k)}, \mathbf{0}, \mathbf{0}, \ldots\right),
$$

where

$$
\boldsymbol{g}_{1, i}^{(k)}=\sum_{j=0}^{u+c k-1} \alpha_{u+c k-j} \boldsymbol{e}_{1} B_{j-i+c}, \quad i=0,1,2, \ldots, c-1,
$$

and

$$
\boldsymbol{g}_{1, i}^{(k)}=\sum_{j=i-c}^{u+c k-1} \alpha_{u+c k-j} \boldsymbol{e}_{1} B_{j-i+c}, i=c, c+1, c+2, \ldots, u+c(k+1)-1 .
$$

Note that $\boldsymbol{g}_{1}^{(k)}$ contains zeros from level $u+c(k+1)$ onward, which is $c$ levels further than that in $\boldsymbol{g}_{0}^{(k)}=\boldsymbol{b}^{(k)}$. Since (2.7) infers $\boldsymbol{g}_{n}^{(k)}=\boldsymbol{g}_{n-1}^{(k)} C$ for $n \in \mathbb{Z}^{+}$, we can continue this process inductively to establish that $\mathbf{g}_{n}^{(k)}$ contains zeros from level $u+c(k+n)$ onward, so that

$$
\boldsymbol{g}_{n}^{(k)}=\left(\boldsymbol{g}_{n, 0}^{(k)}, \boldsymbol{g}_{n, 1}^{(k)}, \boldsymbol{g}_{n, 2}^{(k)}, \ldots, \boldsymbol{g}_{n, u+c(k+n)-2}^{(k)}, \boldsymbol{g}_{n, u+c(k+n)-1}^{(k)}, \mathbf{0}, \mathbf{0}, \ldots\right),
$$

where

$$
\boldsymbol{g}_{n, i}^{(k)}=\left\{\begin{array}{l}
\sum_{j=0}^{u+c(k+n-1)-1} \boldsymbol{g}_{n-1, j}^{(k)} B_{j-i+c} \text { if } i=0,1,2, \ldots, c-1, \\
\sum_{j=i-c}^{u+c(k+n-1)-1} \boldsymbol{g}_{n-1, j}^{(k)} B_{j-i+c} \text { if } i=c, c+1, c+2, \ldots, u+c(k+n)-1 .
\end{array}\right.
$$

Substituting (2.3) into (3.1), the following recursive procedure for computing the $1 \times n_{a}$ row vector $\boldsymbol{g}_{n}^{(k)}, n \in \mathbb{Z}^{+}$, can then be constructed:

$$
\boldsymbol{g}_{n, i}^{(k)}=\left(\sum_{j=0}^{u+c(k+n-1)-1} \alpha_{j-i+c} \boldsymbol{g}_{n-1, j}^{(k)} \boldsymbol{s}, 0,0, \ldots, 0\right), i=0,1,2, \ldots, c-1,
$$


and

$$
\begin{gathered}
\boldsymbol{g}_{n, i}^{(k)}=\boldsymbol{g}_{n-1, i-c}^{(k)} S+\left(\sum_{j=i-c+1}^{u+c(k+n-1)-1} \alpha_{j-i+c} \boldsymbol{g}_{n-1, j}^{(k)} \boldsymbol{s}, 0,0, \ldots, 0\right) \\
i=c, c+1, c+2, \ldots, u+c(k+n)-1,
\end{gathered}
$$

starting with $\boldsymbol{g}_{0, j}^{(k)}=\alpha_{u+c k-j} \boldsymbol{e}_{1}, j=0,1,2, \ldots, u+c k-1$. We remark that further simplifications are possible. In particular, we first note that $\boldsymbol{g}_{n, u+c(k+n)-1}^{(k)}=$ $\boldsymbol{g}_{n-1, u+c(k+n-1)-1}^{(k)} S$ from (3.3). If we repeatedly apply (3.3) to each resulting equation, we eventually obtain that $\boldsymbol{g}_{n, u+c(k+n)-1}^{(k)}=\boldsymbol{g}_{0, u+c k-1}^{(k)} S^{n}$. However, $S$ is of the form (2.1), and one can readily verify that $S^{n}$ becomes an $n_{a} \times n_{a}$ matrix of zeros if $n \geq n_{a}$. Hence, it immediately follows that $\boldsymbol{g}_{n, u+c(k+n)-1}^{(k)}=\mathbf{0}$ if $n \geq n_{a}$. Moreover, it is a straightforward exercise to prove by induction that $\mathbf{g}_{n, u+c(k+n)-j}^{(k)}=$ 0 if $n \geq j n_{a}, j \in \mathbb{Z}^{+}$. The verification of this result is left to the reader.

With the above basic recursive procedure for $\boldsymbol{g}_{n}^{(k)}$ in place, other recursive procedures can readily be established. For example, noting that $\boldsymbol{e}_{1} \boldsymbol{s}=1-\tau_{1}=$ $a_{1},(2.10)$ reduces to give $\psi_{1, i, j}^{(k)}(u)=a_{1} \alpha_{i+j} \alpha_{u+c(k+1)-i}$, and for $n=2,3,4, \ldots$,

$\psi_{n, i, j}^{(k)}(u)=\left\{\begin{array}{c}a_{1} \alpha_{i+j} \sum_{\ell=0}^{u+c(k+n-2)-1} \alpha_{\ell-i+2 c} \boldsymbol{g}_{n-2, \ell}^{(k)} \boldsymbol{s} \\ \text { if } i=c, c+1, \ldots, \min \left\{2 c-1, m_{\alpha}-j\right\}, \\ \alpha_{i+j} \boldsymbol{g}_{n-2, i-2 c}^{(k)} S \boldsymbol{s}+a_{1} \alpha_{i+j} \sum_{\ell=i+1-2 c}^{u+c(k+n-2)-1} \alpha_{\ell-i+2 c} \boldsymbol{g}_{n-2, \ell}^{(k)} \boldsymbol{s} \\ \text { if } i=2 c, 2 c+1, \ldots, m_{\alpha}-j .\end{array}\right.$

In addition, we observe that since $\boldsymbol{g}_{n, u+c k+\ell}^{(k)}=\mathbf{0} \forall \boldsymbol{\ell}=c n, c n+1, c n+2, \ldots$, it follows from (2.3), (2.5), and (2.8) that

$\boldsymbol{h}_{n,-j}^{(k)}=\sum_{\ell=c}^{u+c(k+n)-1} \boldsymbol{g}_{n-1, \ell-c}^{(k)} B_{j+\ell}=\left(\sum_{\ell=c}^{u+c(k+n)-1} \alpha_{j+\ell} \boldsymbol{g}_{n-1, \ell-c}^{(k)} \boldsymbol{s}, 0,0, \ldots, 0\right)$.

Hence, substituting (3.4) into (2.9) immediately yields

$$
\phi_{n, j}^{(k)}(u)=\sum_{\ell=c}^{u+c(k+n)-1} \alpha_{j+\ell} \boldsymbol{g}_{n-1, \ell-c}^{(k)} \boldsymbol{s} .
$$

\section{Numerical EXAMPLes}

In this section, we illustrate the application of our proposed algorithm with several numerical examples. All calculations in this section were carried out on an IBM Pentium IV clone with a $3 \mathrm{Ghz}$ CPU and 4 GB of RAM. 


\section{- Example 1}

Our first example is intended to demonstrate the well-known result that the ordinary and stationary models are identical when interclaim times are geometrically distributed. Specifically, suppose that ordinary interclaim times have pmf

$$
a_{j}= \begin{cases}(0.075)(0.925)^{j-1} & \text { if } j=1,2,3, \ldots, n_{a}-1, \\ (0.925)^{n_{a}-1} & \text { if } j=n_{a} .\end{cases}
$$

In other words, the pmf (4.1) is that of a truncated geometric distribution with all the probability mass on $\left\{n_{a}, n_{a}+1, n_{a}+2, \ldots\right\}$ assigned to the support value $n_{a}$. Clearly, $\sum_{j=1}^{n_{a}} a_{j}=1$. Moreover, as $n_{a}$ becomes larger, the closer $\left\{a_{j}\right\}_{j=1}^{n_{a}}$ approximates this particular geometric distribution having mean $40 / 3 \simeq 13.333$. Let the individual claim amount distribution be given by the pmf

$$
a_{j}=G(j-1)-G(j), j \in \mathbb{Z}^{+},
$$

where $G(x)=(1+x / 30)^{-4}, x \geq 0$, is the survival function of a Pareto distribution having mean 10 . Note that $m_{\alpha}=\infty$, which implies that both $\left|U_{T}\right|$ and $U_{T-}$ are distributed on $\mathbb{Z}^{+}$if $c=1$.

Table 1 displays the values (rounded to 5 decimal places) of

$$
\Psi_{n, x, y}(u)=\operatorname{Pr}\left\{T<n, U_{T-} \leq x,\left|U_{T}\right| \leq y \mid U_{0}=u\right\}=\sum_{\ell=1}^{n-1} \sum_{i=c}^{x} \sum_{j=1}^{y} \psi_{\ell, i, j}(u)
$$

for a discrete-time risk process with $u=50$ and $c=1$ having the above interclaim time and claim amount distributions. The values in Table 1 were generated by first implementing the recursive procedures (3.2) and (3.3) using Mathematica (Version 5), and then summing the trivariate probabilities computed via (2.10) and (2.16). However, we point out that (2.15) and (3.5) were employed to calculate the joint probabilities when $x=\infty$. Similarly, (2.17) was used in the case where $y=\infty$. The key to Table 1 is as follows:

(1) ordinary model with $n_{a}=10$ (so that $\sum_{j=1}^{n_{a}-1} a_{j}=0.504$ and $a_{n_{a}}=0.496$ );

(2) stationary model with $n_{a}=10$;

(3) ordinary model with $n_{a}=25$ (so that $\sum_{j=1}^{n_{a}-1} a_{j}=0.846$ and $a_{n_{a}}=0.154$ );

(4) stationary model with $n_{a}=25$;

(5) ordinary model with $n_{a}=50$ (so that $\sum_{j=1}^{n_{a}-1} a_{j}=0.978$ and $a_{n_{a}}=0.022$ );

(6) stationary model with $n_{a}=50$.

We make the following observations concerning the results in Table 1:

(a) When $n_{a}=10$, the mean (ordinary) interclaim time is 7.219 , which happens to be less than the claim amount mean. Consequently, as ruin is certain to 
occur, the values in Table 1 when $n_{a}=10$ approach 1 as $n, x$, and $y$ grow larger.

(b) When $n_{a}=25$ and 50, the mean (ordinary) interclaim times are 11.435 and 13.063 respectively, which are both greater than the claim amount mean. As a result, the probability of ultimate ruin is strictly less than 1 . This fact is reflected in the values in Table 1 , as the probabilities for $n_{a}=25$ and 50 are significantly smaller when compared to their corresponding counterparts for $n_{a}=10$.

(c) Under the stationary model assumption, the mean (initial) interclaim times are $4.863,9.182$, and 12.298 for $n_{a}=10,25$, and 50 respectively. Note that these means are smaller than their corresponding counterparts in the ordinary model. As a result, ruin is more likely to occur on the first claim under the stationary model than under the ordinary one. For this reason, the values of $\Psi_{n, x, y}(50)$ under the stationary model are always larger than the corresponding values in the ordinary model. However, Table 1 indicates that the difference between the two models becomes less and less as $n_{a}$ grows from 10 to 25 and finally to 50 , as expected.

\section{- Example 2}

Our second example has been chosen to illustrate, for comparative purposes, the same computations carried out in our first example, but for a more advanced model than the compound binomial one. In particular, we now assume that the ordinary interclaim times have pmf

$$
a_{j}= \begin{cases}\frac{4}{15}(0.3)(0.7)^{j-1}+\frac{19}{30}(0.075)(0.925)^{j-1}+\frac{1}{10}(0.025)(0.975)^{j-1} & \text { if } j=1,2,3, \ldots, n_{a}-1 \\ \frac{4}{15}(0.7)^{n_{a}-1}+\frac{19}{30}(0.925)^{n_{a}-1}+\frac{1}{10}(0.975)^{n_{a}-1} & \text { if } j=n_{a} .\end{cases}
$$

We recognize the pmf (4.4) as that from a truncated (at $\left.n_{a}\right) 3$-point mixture of geometric distributions having overall mean $(4 / 15)(10 / 3)+(19 / 30)(40 / 3)+$ $(1 / 10)(40)=40 / 3$. We remark that this mean is identical to the ordinary interclaim time mean considered in our first example. In what follows, we fix $n_{a}=60$ so that $\sum_{j=1}^{n_{a}-1} a_{j}=0.971$ and $\sum_{j=1}^{n_{a}} j a_{j}=12.379$. Moreover, we assume that the pmf of the individual claim amount distribution is once again defined by (4.2).

Table 2 displays the values (rounded to 5 decimal places) of $\Psi_{n, x, y}(50)$ for several variants of a discrete-time risk process (again with $c=1$ ) involving the above interclaim time and claim amount distributions, namely:

(1) ordinary model;

(2) stationary model;

(3) delayed model where $W_{1}$ is a truncated (at $n_{r}=200$ ) 3-point mixture of geometric distributions with pmf 


$$
r_{j}= \begin{cases}\frac{1}{15}(0.3)(0.7)^{j-1}+\frac{19}{30}(0.075)(0.925)^{j-1}+\frac{3}{10}(0.025)(0.975)^{j-1} & \text { if } j=1,2,3, \ldots, 199, \\ \frac{1}{15}(0.7)^{199}+\frac{19}{30}(0.925)^{199}+\frac{3}{10}(0.975)^{199} & \text { if } j=200\end{cases}
$$

(4) delayed model where $W_{1}$ is truncated (at $n_{r}=50$ ) geometric with pmf

$$
r_{j}= \begin{cases}(0.075)(0.925)^{j-1} & \text { if } j=1,2,3, \ldots, 49, \\ (0.925)^{49} & \text { if } j=50\end{cases}
$$

(5) delayed model where $W_{1}$ is discrete uniform with $\operatorname{pmf} r_{j}=0.04, j=1,2,3$, ..., 25;

(6) delayed model where $W_{1}=1$ with probability 1 .

We make the following comments about Table 2:

(a) Comparison of all values of $\Psi_{n, x, y}(50)$ corresponding to entry (5) of Table 1 and entry (1) of Table 2 seem to indicate that ruin is more likely to occur when interclaim times have the 3-point mixture distribution of our second example as opposed to the geometric distribution introduced in our first example. This behaviour is attributable to the values of the mixing weights $\{4 / 15,19 / 30,1 / 10\}$, since the probability of observing a "short" interclaim time (i.e., one that is less than the mean 40/3) is 0.696 under the mixture model and only 0.637 under the geometric model. Conversely, there is less of a difference between the two models in observing a "long" interclaim time (i.e., one that is greater than 30) - specifically, 0.096 under the geometric model and 0.108 under the mixture model.

(b) Under the stationary model assumption with $n_{a}=60$, the mean (initial) interclaim time is 14.721 which is larger than the ordinary interclaim time mean 12.379. Consequently, ruin is more likely to occur later in the stationary model than in the ordinary model. Hence, the values of $\Psi_{n, x, y}(50)$ under the stationary model are consistently smaller than the corresponding values in the ordinary model.

(c) It is not a difficult exercise to show that if the ordinary interclaim times are distributed according to the non-truncated (i.e., infinite support) version of the 3-point mixture of geometrics distribution given by (4.4), then the limiting form of the forward recurrence time distribution is again a 3point mixture of the same geometrics but with different weights, namely $\{1 / 15,19 / 30,3 / 10\}$ instead of $\{4 / 15,19 / 30,1 / 10\}$. This new distribution has mean $62 / 3 \simeq 20.667$, and in fact represents the true distribution for $W_{1}$ in the stationary model. Being a longer-tailed distribution, however, it is therefore not surprising that using $n_{a}=60$ in model (2) is somewhat inadequate since a mean (initial) interclaim time of 14.721 is only achieved. As a result, we propose model (3) with $n_{r}=200$ as a more accurate reflection of the stationary model since $\sum_{j=1}^{n_{r}} j r_{j}=20.250$. 
(d) At first glance, it would appear that the ranking of models (1) through (5) based on their values of $\Psi_{n, x, y}(50)$ is identical to the ranking of models based on their mean (initial) interclaim time values. The mean (initial) interclaim times corresponding to delayed models (4) and (5) are 13.063 and 13 respectively, both of which lie between the means corresponding to models (1) and (2). A notable exception occurs when $n=50$, however, as values of $\Psi_{n, x, y}(50)$ for model (5) are always smaller (albeit slightly) than their corresponding counterparts for model (2). Even with that said, the values of $\Psi_{n, x, y}(50)$ look quite similar across models (1) through (5).

(e) In the absence of any knowledge regarding the choice of distribution to employ for the first interclaim time, model (6) can be viewed as the "worstcase scenario" model, thereby serving to provide upper bounds on the values of $\Psi_{n, x, y}(50)$.

\section{- Example 3}

Our final example is intended to show how our discrete-time model can be used to approximate ruin-related quantities in the analogous continuous-time delayed Sparre Andersen model. Specifically, let $\mathcal{U}(t)=v+\xi t-\sum_{i=1}^{\mathcal{X}(t)} Y_{i}, t \geq 0$, represent a continuous-time surplus process where:

- $v \geq 0$ is the insurer's initial surplus,

- $\xi$ is the insurer's premium rate per unit time,

- $\mathcal{N}(t)$ is the number of claims in the time interval $(0, t]$,

- $\left\{Y_{1}, \mathscr{Y}_{2}, \mathscr{Y}_{3}, \ldots\right\}$ is a sequence of iid positive claim amount random variables with common survival function $G(y), y \geq 0$.

If $\left\{\mathcal{W}_{1}, \mathcal{W}_{2}, \mathcal{W}_{3}, \ldots\right\}$ denotes the corresponding sequence of independent positive interclaim time random variables, where $\mathcal{W}_{1}$ is the duration from time 0 until the first claim occurs and $\mathcal{W}_{i}, i=2,3,4, \ldots$, is the time between the $(i-1)$-th and $i$-th claims, then it follows that $\mathcal{N}(t)=\max \left\{k: \mathcal{W}_{1}+\mathcal{W}_{2}+\cdots+\mathcal{W}_{k} \leq t\right\}$. For $w \geq 0$, define $K_{1}(w)=\operatorname{Pr}\left\{\mathcal{W}_{1}>w\right\}$ and $K(w)=\operatorname{Pr}\left\{\mathcal{W}_{i}>w\right\}, i=2,3,4, \ldots$ Moreover, let $\mathcal{T}=\inf \{t>0: \mathcal{U}(t)<0\}$ represent the time of ruin random variable with $\mathcal{T}=\infty$ if $\mathcal{U}(t) \geq 0 \forall t>0$.

We essentially follow the ideas in Dickson et al. (1995) to construct a discrete-time approximation to the above surplus process. First of all, we rescale the above process by multiplying all monetary amounts by a parameter $\beta>0$ and taking a new time unit to be $\kappa^{-1}$ times the original time unit where $\kappa>0$ is another parameter. We remark that $\beta$ and $\kappa$ should be suitably chosen so that the rescaled initial reserve (i.e., $\beta v$ ) and premium rate per unit time (i.e., $\beta \xi / \kappa$ ) are both positive integers.

In order to discretize the rescaled claim amount distribution, define $\left\{Y_{1}^{(d)}\right.$, $\left.Y_{2}^{(d)}, Y_{3}^{(d)}, \ldots\right\}$ to be the sequence of iid random variables whose distribution is approximately the same as that of $\beta Y_{1}$ and which are distributed on $\mathbb{Z}^{+}$. As in the previous two examples, one particular choice is to use 


$$
\alpha_{j}^{(d)}=G\left(\frac{j-1}{\beta}\right)-G\left(\frac{j}{\beta}\right), j \in \mathbb{Z}^{+},
$$

as the approximating claim amount pmf (this is the "lower bound" discretization method discussed in Dickson (2005, p. 79)).

We next introduce the rescaled number of claims process

$$
\mathcal{N}_{\kappa}(t)=\mathcal{N}(t / \kappa)=\max \left\{k: \mathcal{W}_{\kappa, 1}+\mathcal{W}_{\kappa, 2}+\cdots+\mathcal{W}_{\kappa, k} \leq t,\right\},
$$

where $\mathcal{W}_{\kappa, i}=\kappa \mathcal{W}_{i} \forall i \in \mathbb{Z}^{+}$. In order to apply our proposed methodology, we must now discretize the distributions of $\mathcal{W}_{\kappa, 1}$ and $\mathcal{W}_{\kappa, 2}$ on intervals of finite support so as to create an approximating modified discrete-time renewal process $\left\{N_{d}(t): t=0,1,2, \ldots\right\}$. For specified (small) tolerances of $\varepsilon_{1}>0$ and $\varepsilon_{2}>0$, one can select support values $n_{r}$ and $n_{a}$ so that

$$
n_{r}=\min \left\{n \in \mathbb{Z}^{+}: K_{1}(n / \kappa) \leq \varepsilon_{1}\right\}
$$

and

$$
n_{a}=\min \left\{n \in \mathbb{Z}^{+}: K(n / \kappa) \leq \varepsilon_{2}\right\} .
$$

Once $n_{r}$ and $n_{a}$ have been suitably chosen, one can then construct

$$
r_{j}= \begin{cases}K_{1}\left(\frac{j-1}{\kappa}\right)-K_{1}\left(\frac{j}{\kappa}\right) & \text { if } j=1,2,3, \ldots, n_{r}-1, \\ K_{1}\left(\frac{n_{r}-1}{\kappa}\right) & \text { if } j=n_{r},\end{cases}
$$

and

$$
a_{j}= \begin{cases}K\left(\frac{j-1}{\kappa}\right)-K\left(\frac{j}{\kappa}\right) & \text { if } j=1,2,3, \ldots, n_{a}-1, \\ K\left(\frac{n_{a}-1}{\kappa}\right) & \text { if } j=n_{a},\end{cases}
$$

as the approximating pmf's of $\mathcal{W}_{\kappa, 1}$ and $\mathcal{W}_{\kappa, 2}$ respectively.

If we now consider the discrete-time surplus process

$$
\mathcal{U}_{d}(t)=\beta v+(\beta \xi / \kappa) t-\sum_{i=1}^{N_{d}(t)} Y_{i}^{(d)}, t=0,1,2, \ldots
$$

then it is clear from the above construction that $\mathcal{U}_{d}(\kappa t) / \beta$ will have approximately the same distribution as $\mathcal{U}(t)$. In fact, increasing the value of $\kappa$ should result in an improved approximation.

To demonstrate the effectiveness of our approximation procedure, we consider the Erlang-2 example in Dickson and Willmot (2005, pp. 58-59) concerning a compound Poisson risk model in which $\xi=1$, 


$$
K_{1}(w)=K(w)=e^{-w}, w \geq 0
$$

and

$$
G(y)=(2 y+1) e^{-2 y}, y \geq 0 \text {. }
$$

Table 3 displays values (rounded to 4 decimal places) for both the exact and approximated finite-time ruin probabilities (i.e., $\operatorname{Pr}\{\mathcal{T} \leq t \mid \mathcal{U}(0)=v\}$ ) under this model for $v=1,10$ and $t=2,4,6,8,10,20,40$. The exact values are taken directly from Table 1 of Dickson and Willmot (2005, p. 59). The approximated values were obtained by applying the above discretization procedure using $\beta=20$ and $\kappa=22$ (so as to yield a rescaled premium rate of 1 ) and then appropriately summing the following univariate pmf (with $u=\beta v$ )

$$
\sigma_{n}(u)=\operatorname{Pr}\left\{T=n \mid \mathcal{U}_{d}(0)=u\right\}=\sum_{j=1}^{m_{\alpha}-c} \phi_{n, j}(u), n \in \mathbb{Z}^{+},
$$

which simplifies (via (2.15) and (3.5)) to give

$$
\begin{aligned}
\sigma_{n}(u)= & \sum_{i=c}^{u+c n-1}\left(\Lambda_{i}-\Lambda_{i+m_{\alpha}-c}\right) \sum_{k=1}^{\min \left\{n-1, n_{r}\right\}} r_{k}\left(\boldsymbol{g}_{n-k-1, i-c}^{(k)} \boldsymbol{s}\right) \\
& +r_{n}\left(\Lambda_{u+c n}-\Lambda_{u+c}(n-1)+m_{\alpha}\right)
\end{aligned}
$$

In selecting the value of $n_{a}\left(=n_{r}\right)$, we remark that a tolerance level of $2.1 \%$ was used which resulted in $n_{a}=85$. The values under the heading "Approx. 1 " in Table 3 correspond to the definition of the time of ruin given in Section 1, namely $T=\min \left\{t \in \mathbb{Z}^{+}: \mathcal{U}_{d}(t)<0\right\}$ (i.e., a surplus of zero is not viewed as ruin). Conversely, the values under the heading "Approx. 2" correspond to a slightly altered definition of the time of ruin random variable, namely $T^{*}=$ $\min \left\{t \in \mathbb{Z}^{+}: \mathcal{U}_{d}(t) \leq 0\right\}$ (i.e., a surplus of zero is regarded as ruin). Clearly, $\operatorname{Pr}\left\{T^{*}=n \mid \mathcal{U}_{d}(0)=u\right\}=\sigma_{n}(u-1)$ for $u \in \mathbb{Z}^{+}$. Dickson et al. (1995) discuss the use of these two definitions in approximating continuous-time ruin-related quantities of interest, and we include both of them here for comparative purposes. All in all, Table 3 demonstrates that our proposed method yields decent approximations even for a moderate choice of $\kappa=22$. Based on the accuracy in the table, however, we expect the approximation to improve for larger values of $\kappa$.

In fact, if we make further use of the relations in Dickson et al. (1995, p. 171), we can readily establish that the joint probability distribution of the time of ruin, the surplus immediately prior to ruin, and the deficit at ruin in the continuous-time delayed Sparre Andersen model, namely

$$
\operatorname{Pr}\left\{\mathcal{T}<t, \mathcal{U}\left(\mathcal{T}_{-}\right) \leq x,|\mathcal{U}(\mathcal{T})| \leq y \mid \mathcal{U}(0)=v\right\}, t>0 ; x>0 ; y>0
$$


can be approximated by the quantity $\Psi_{\kappa t, \beta x+\beta \xi / \kappa-1, \beta y}(\beta v-1)$ defined by (4.3). Applying the same discretization procedure described above, Table 4 displays approximated values (rounded to 5 decimal places) of $\operatorname{Pr}\{\mathcal{T}<t, \mathcal{U}(\mathcal{T}-) \leq x$, $|\mathcal{U}(\mathcal{T})| \leq y \mid \mathcal{U}(0)=v\}$ corresponding to the Dickson and Willmot (2005) Erlang-2 example for $v=1$. However, we remark that we are only able to compare the approximated values to their exact counterparts when $x=\infty$. In this case, exact probabilities of the bivariate distribution of the time of ruin and the deficit at ruin can be calculated by means of the approach described in Dickson and Drekic (2006). The exact probabilities are italicized and appear in parentheses next to their approximated values under the heading " $x=\infty$ " in Table 4. Based on our findings here, it would appear that our proposed approach provides accurate approximations to these joint ruin probabilities.

\section{ACKNOWLEDGEMENTS}

The authors would like to thank the anonymous referee for the useful comments and suggestions which helped to improve this paper. The authors would also like to thank Ana Maria Mera for her valuable programming assistance. This work has been assisted by the Natural Sciences and Engineering Research Council of Canada.

\section{REFERENCES}

ALFA, A.S. (2004) "Markov chain representations of discrete distributions applied to queueing models". Computers \& Operations Research 31, 2365-2385.

Alfa, A.S. and NeUTS, M.F. (1995) "Modelling vehicular traffic using the discrete time Markovian arrival process". Transportation Science 29, 109-117.

Cardoso, R.M.R. and Egidio dos Reis, A.D. (2002) "Recursive calculation of time to ruin distributions". Insurance: Mathematics \& Economics 30, 219-230.

Cheng, S., Gerber, H.U. and Shiu, E.S.W. (2000) "Discounted probabilities and ruin theory in the compound binomial model". Insurance: Mathematics \& Economics 26, 239-250.

Cossette, H., Landriault, D. and Marceau, E. (2006) "Ruin probabilities in the discrete time renewal risk model". Insurance: Mathematics \& Economics 38, 309-323.

De Vylder, F.E. and Marceau, E. (1996) "Classical numerical ruin probabilities". Scandinavian Actuarial Journal, 109-123.

DiCKSON, D.C.M. (1994) "Some comments on the compound binomial model". ASTIN Bulletin 24, 33-45.

Dickson, D.C.M. (2005) Insurance Risk and Ruin, Cambridge University Press, Cambridge.

Dickson, D.C.M. and DREKIC, S. (2006) "Optimal dividends under a ruin probability constraint". Annals of Actuarial Science 1, 291-306.

Dickson, D.C.M., Egidio dos Reis, A.D. and Waters, H.R. (1995) "Some stable algorithms in ruin theory and their applications". ASTIN Bulletin 25, 153-175.

DiCKSON, D.C.M. and WATERS, H.R. (1991) "Recursive calculation of survival probabilities". ASTIN Bulletin 21, 199-221.

Dickson, D.C.M. and Willmot, G.E. (2005) "The density of the time to ruin in the classical Poisson risk model". ASTIN Bulletin 35, 45-60.

Gerber, H.U. (1988) "Mathematical fun with the compound binomial process". ASTIN Bulletin 18, 161-168. 
Gerber, H.U. and ShiU, E.S.W. (1998) "On the time value of ruin". North American Actuarial Journal 2(1), 48-78.

Karlin, S. and TAYlor, H.M. (1975) A First Course in Stochastic Processes, 2nd edition, Academic Press, New York.

LI, S. (2005a) "On a class of discrete time renewal risk models". Scandinavian Actuarial Journal, 241-260.

LI, S. (2005b) "Distributions of the surplus before ruin, the deficit at ruin and the claim causing ruin in a class of discrete time risk models". Scandinavian Actuarial Journal, 271-284.

LI, S. and GARRIDO, J. (2002) "On the time value of ruin in the discrete time risk model". Business Economics Series 12, Working Paper 02-18, Universidad Carlos III de Madrid, Spain, $28 \mathrm{pp}$.

MicheL, R. (1989) "Representation of a time-discrete probability of eventual ruin". Insurance: Mathematics \& Economics 8, 149-152.

Pavlova, K.P. and Willmot, G.E. (2004) "The discrete stationary renewal risk model and the Gerber-Shiu discounted penalty function”. Insurance: Mathematics \& Economics 35, 267277.

SHIU, E.S.W. (1989) "Probability of eventual ruin in the compound binomial model". ASTIN Bulletin 19, 179-190.

WiLlmot, G.E. (1993) "Ruin probabilities in the compound binomial model". Insurance: Mathematics \& Economics 12, 133-142.

Attahiru Sule Alfa

Department of Electrical and Computer Engineering

University of Manitoba

$75 \mathrm{~A}$ Chancellor's Circle

Winnipeg, Manitoba, Canada R3T 5 V6

E-mail: alfa@ee.umanitoba.ca

Steve Drekic (corresponding author)

Department of Statistics and Actuarial Science

University of Waterloo

200 University Ave. West

Waterloo, Ontario, Canada N2L $3 G 1$

E-mail:sdrekic@math.uwaterloo.ca 
TABLE 1

VALUES OF $\Psi_{n, x, y}(50)$ CORRESPONDING TO EXAMPLE 1

(a) $x=10$

\begin{tabular}{|c|c|c|c|c|c|}
\hline & & $n=50$ & $n=100$ & $n=250$ & $n=500$ \\
\hline \multirow[t]{6}{*}{$y=10$} & (1) & 0.01766 & 0.04572 & 0.09105 & 0.10791 \\
\hline & (2) & 0.02067 & 0.04936 & 0.09288 & 0.10846 \\
\hline & (3) & 0.00677 & 0.01373 & 0.02580 & 0.03458 \\
\hline & (4) & 0.00755 & 0.01483 & 0.02706 & 0.03580 \\
\hline & (5) & 0.00630 & 0.01173 & 0.01982 & 0.02473 \\
\hline & (6) & 0.00643 & 0.01194 & 0.02009 & 0.02501 \\
\hline \multirow[t]{6}{*}{$y=25$} & (1) & 0.02490 & 0.06452 & 0.12852 & 0.15232 \\
\hline & (2) & 0.02915 & 0.06965 & 0.13110 & 0.15310 \\
\hline & (3) & 0.00953 & 0.01934 & 0.03633 & 0.04870 \\
\hline & (4) & 0.01063 & 0.02088 & 0.03812 & 0.05043 \\
\hline & (5) & 0.00887 & 0.01652 & 0.02791 & 0.03483 \\
\hline & (6) & 0.00905 & 0.01681 & 0.02829 & 0.03522 \\
\hline \multirow[t]{6}{*}{$y=50$} & (1) & 0.02752 & 0.07134 & 0.14212 & 0.16846 \\
\hline & (2) & 0.03222 & 0.07701 & 0.14498 & 0.16932 \\
\hline & (3) & 0.01052 & 0.02136 & 0.04013 & 0.05380 \\
\hline & (4) & 0.01174 & 0.02307 & 0.04210 & 0.05570 \\
\hline & (5) & 0.00979 & 0.01825 & 0.03083 & 0.03847 \\
\hline & (6) & 0.00999 & 0.01857 & 0.03124 & 0.03890 \\
\hline \multirow[t]{6}{*}{$y=\infty$} & (1) & 0.02843 & 0.07370 & 0.14684 & 0.17405 \\
\hline & (2) & 0.03328 & 0.07957 & 0.14980 & 0.17494 \\
\hline & (3) & 0.01086 & 0.02205 & 0.04144 & 0.05555 \\
\hline & (4) & 0.01212 & 0.02382 & 0.04347 & 0.05751 \\
\hline & (5) & 0.01011 & 0.01884 & 0.03184 & 0.03973 \\
\hline & (6) & 0.01032 & 0.01917 & 0.03226 & 0.04017 \\
\hline
\end{tabular}

(b) $x=25$

\begin{tabular}{llcccc}
\hline \hline & & $n=50$ & $n=100$ & $n=250$ & $n=500$ \\
\hline$y=10$ & $(1)$ & 0.04981 & 0.13476 & 0.27343 & 0.32501 \\
& $(2)$ & 0.05876 & 0.14587 & 0.27914 & 0.32683 \\
& $(3)$ & 0.01673 & 0.03494 & 0.06645 & 0.08921 \\
& $(4)$ & 0.01872 & 0.03779 & 0.06977 & 0.09242 \\
& $(5)$ & 0.01561 & 0.02979 & 0.05080 & 0.06343 \\
& $(6)$ & 0.01593 & 0.03033 & 0.05148 & 0.06414 \\
$y=25$ & $(1)$ & 0.07384 & 0.20002 & 0.40604 & 0.48268 \\
& $(2)$ & 0.08714 & 0.21653 & 0.41454 & 0.48539 \\
& $(3)$ & 0.02471 & 0.05168 & 0.09834 & 0.13203 \\
& (4) & 0.02765 & 0.05591 & 0.10325 & 0.13679
\end{tabular}




\begin{tabular}{cccccc}
\hline \hline & & $n=50$ & $n=100$ & $n=250$ & $n=500$ \\
\hline & $(5)$ & 0.02306 & 0.04406 & 0.07515 & 0.09384 \\
& $(6)$ & 0.02353 & 0.04485 & 0.07617 & 0.09490 \\
$y=50$ & (1) & 0.08409 & 0.22792 & 0.46278 & 0.55016 \\
& (2) & 0.09924 & 0.24674 & 0.47248 & 0.55324 \\
& (3) & 0.02809 & 0.05878 & 0.11188 & 0.15022 \\
& (4) & 0.03144 & 0.06359 & 0.11748 & 0.15564 \\
& $(5)$ & 0.02621 & 0.05011 & 0.08549 & 0.10675 \\
& $(6)$ & 0.02675 & 0.05101 & 0.08665 & 0.10795 \\
$y=\infty$ & $(1)$ & 0.08838 & 0.23962 & 0.48661 & 0.57849 \\
& $(2)$ & 0.10431 & 0.25941 & 0.49681 & 0.58174 \\
& (3) & 0.02949 & 0.06174 & 0.11754 & 0.15782 \\
& (4) & 0.03301 & 0.06680 & 0.12342 & 0.16352 \\
& (5) & 0.02752 & 0.05264 & 0.08980 & 0.11214 \\
& (6) & 0.02809 & 0.05358 & 0.09102 & 0.11340 \\
\hline & & & & &
\end{tabular}

(c) $x=50$

\begin{tabular}{llllll}
\hline \hline & & $n=50$ & $n=100$ & $n=250$ & $n=500$ \\
\hline$y=10$ & $(1)$ & 0.07605 & 0.19299 & 0.37938 & 0.44862 \\
& $(2)$ & 0.08953 & 0.20881 & 0.38767 & 0.45164 \\
& $(3)$ & 0.02715 & 0.05581 & 0.10524 & 0.14077 \\
& $(4)$ & 0.03044 & 0.06049 & 0.11066 & 0.14603 \\
& $(5)$ & 0.02514 & 0.04706 & 0.07935 & 0.09865 \\
& $(6)$ & 0.02566 & 0.04791 & 0.08044 & 0.09978 \\
$y=25$ & $(1)$ & 0.11790 & 0.29742 & 0.58288 & 0.68889 \\
& $(2)$ & 0.13878 & 0.32180 & 0.59567 & 0.69362 \\
& $(3)$ & 0.04225 & 0.08673 & 0.16340 & 0.21848 \\
& $(4)$ & 0.04739 & 0.09403 & 0.17185 & 0.22669 \\
& $(5)$ & 0.03910 & 0.07307 & 0.12308 & 0.15294 \\
& $(6)$ & 0.03991 & 0.07439 & 0.12477 & 0.15469 \\
$y=50$ & $(1)$ & 0.13873 & 0.34836 & 0.68107 & 0.80461 \\
& $(2)$ & 0.16328 & 0.37691 & 0.69608 & 0.81023 \\
& $(3)$ & 0.04988 & 0.10227 & 0.19253 & 0.25735 \\
& $(4)$ & 0.05595 & 0.11181 & 0.20250 & 0.26705 \\
& $(5)$ & 0.04615 & 0.08612 & 0.14492 & 0.18001 \\
& $(6)$ & 0.04710 & 0.08767 & 0.14691 & 0.18208 \\
$y=\infty$ & $(1)$ & 0.14965 & 0.37429 & 0.73027 & 0.86244 \\
& $(2)$ & 0.17611 & 0.40496 & 0.74642 & 0.86854 \\
& $(3)$ & 0.05397 & 0.11051 & 0.20791 & 0.27784 \\
& $(4)$ & 0.06055 & 0.11984 & 0.21870 & 0.28833 \\
& $(5)$ & 0.04992 & 0.09303 & 0.15643 & 0.19425 \\
& $(6)$ & 0.05095 & 0.09471 & 0.15858 & 0.19649
\end{tabular}


(d) $x=\infty$

\begin{tabular}{llllll}
\hline \hline & & $n=50$ & $n=100$ & $n=250$ & $n=500$ \\
\hline$y=10$ & (1) & 0.09293 & 0.21747 & 0.41323 & 0.48604 \\
& (2) & 0.10716 & 0.23343 & 0.42092 & 0.48816 \\
& (3) & 0.03848 & 0.07360 & 0.13466 & 0.17977 \\
& (4) & 0.04269 & 0.07923 & 0.14105 & 0.18593 \\
& (5) & 0.03549 & 0.06271 & 0.10375 & 0.12918 \\
& (6) & 0.03623 & 0.06380 & 0.10511 & 0.13059 \\
$y=25$ & (1) & 0.14843 & 0.34182 & 0.64440 & 0.75697 \\
& (2) & 0.17055 & 0.36630 & 0.65595 & 0.75990 \\
& (3) & 0.06286 & 0.11933 & 0.21778 & 0.29097 \\
& (4) & 0.06963 & 0.12828 & 0.22790 & 0.30072 \\
& (5) & 0.05791 & 0.10176 & 0.16823 & 0.20980 \\
& (6) & 0.05911 & 0.10350 & 0.17042 & 0.21206 \\
$y=50$ & (1) & 0.17911 & 0.40721 & 0.76280 & 0.89514 \\
& (2) & 0.20514 & 0.43572 & 0.77596 & 0.89814 \\
& (3) & 0.07730 & 0.14594 & 0.26604 & 0.35599 \\
& (4) & 0.08548 & 0.15668 & 0.27815 & 0.36761 \\
& (5) & 0.07114 & 0.12455 & 0.20609 & 0.25761 \\
& (6) & 0.07262 & 0.12667 & 0.20873 & 0.26033 \\
$y=\infty$ & (1) & 0.19816 & 0.44527 & 0.82920 & 0.97217 \\
& (2) & 0.22618 & 0.47560 & 0.84277 & 0.97474 \\
& (3) & 0.08721 & 0.16407 & 0.29952 & 0.40236 \\
& (4) & 0.09624 & 0.17582 & 0.31271 & 0.41495 \\
& (5) & 0.08018 & 0.14020 & 0.23300 & 0.29289 \\
& (6) & 0.08184 & 0.14254 & 0.23591 & 0.29590 \\
\hline \hline & & & & &
\end{tabular}

TABLE 2

VALUES OF $\Psi_{n, x, y}(50)$ CORRESPONDING TO EXAMPLE 2

(a) $x=10$

\begin{tabular}{llllll}
\hline \hline & & $n=50$ & $n=100$ & $n=250$ & $n=500$ \\
\hline$y=10$ & (1) & 0.01414 & 0.02473 & 0.04003 & 0.04971 \\
& (2) & 0.01236 & 0.02270 & 0.03801 & 0.04782 \\
& (3) & 0.01150 & 0.02115 & 0.03580 & 0.04543 \\
& (4) & 0.01298 & 0.02360 & 0.03906 & 0.04885 \\
& (5) & 0.01195 & 0.02281 & 0.03852 & 0.04841 \\
& (6) & 0.02221 & 0.03397 & 0.04938 & 0.05857 \\
$y=25$ & (1) & 0.01987 & 0.03474 & 0.05624 & 0.06985 \\
& (2) & 0.01736 & 0.03189 & 0.05341 & 0.06719 \\
& (3) & 0.01615 & 0.02972 & 0.05029 & 0.06384
\end{tabular}




\begin{tabular}{cccccc}
\hline \hline & & $n=50$ & $n=100$ & $n=250$ & $n=500$ \\
\hline \multirow{5}{*}{$y=50$} & (4) & 0.01823 & 0.03316 & 0.05488 & 0.06863 \\
& (5) & 0.01678 & 0.03205 & 0.05412 & 0.06801 \\
& (6) & 0.03121 & 0.04773 & 0.06939 & 0.08229 \\
& (1) & 0.02191 & 0.03832 & 0.06205 & 0.07706 \\
& (2) & 0.01915 & 0.03518 & 0.05892 & 0.07412 \\
& (3) & 0.01782 & 0.03278 & 0.05549 & 0.07043 \\
& (4) & 0.02010 & 0.03658 & 0.06055 & 0.07572 \\
& (5) & 0.01851 & 0.03535 & 0.05970 & 0.07503 \\
& (6) & 0.03442 & 0.05266 & 0.07655 & 0.09079 \\
& & & & & \\
& (1) & 0.02261 & 0.03955 & 0.06404 & 0.07953 \\
& (2) & 0.01976 & 0.03630 & 0.06081 & 0.07650 \\
& (3) & 0.01838 & 0.03383 & 0.05726 & 0.07268 \\
& (4) & 0.02075 & 0.03775 & 0.06248 & 0.07814 \\
& (5) & 0.01910 & 0.03648 & 0.06161 & 0.07743 \\
(6) & 0.03552 & 0.05435 & 0.07900 & 0.09369 \\
\hline
\end{tabular}

(b) $x=25$

\begin{tabular}{llllll}
\hline \hline & & $n=50$ & $n=100$ & $n=250$ & $n=500$ \\
\hline$y=10$ & $(1)$ & 0.03083 & 0.05452 & 0.08857 & 0.10997 \\
& $(2)$ & 0.02686 & 0.04996 & 0.08402 & 0.10569 \\
& $(3)$ & 0.02499 & 0.04655 & 0.07910 & 0.10039 \\
& $(4)$ & 0.02820 & 0.05197 & 0.08636 & 0.10799 \\
& $(5)$ & 0.02584 & 0.05015 & 0.08509 & 0.10694 \\
& $(6)$ & 0.04889 & 0.07534 & 0.10968 & 0.12998 \\
$y=25$ & $(1)$ & 0.04528 & 0.08011 & 0.13016 & 0.16159 \\
& $(2)$ & 0.03944 & 0.07340 & 0.12347 & 0.15530 \\
& $(3)$ & 0.03669 & 0.06839 & 0.11623 & 0.14752 \\
& $(4)$ & 0.04141 & 0.07636 & 0.12690 & 0.15868 \\
& $(5)$ & 0.03793 & 0.07367 & 0.12503 & 0.15714 \\
& $(6)$ & 0.07183 & 0.11073 & 0.16120 & 0.19104 \\
$y=50$ & $(1)$ & 0.05129 & 0.09077 & 0.14749 & 0.18311 \\
& $(2)$ & 0.04467 & 0.08316 & 0.13991 & 0.17598 \\
& $(3)$ & 0.04155 & 0.07748 & 0.13170 & 0.16715 \\
& $(4)$ & 0.04690 & 0.08651 & 0.14380 & 0.17980 \\
& $(5)$ & 0.04295 & 0.08346 & 0.14167 & 0.17806 \\
& $(6)$ & 0.08139 & 0.12549 & 0.18269 & 0.21650 \\
$y=\infty$ & $(1)$ & 0.05375 & 0.09514 & 0.15459 & 0.19193 \\
& $(2)$ & 0.04681 & 0.08716 & 0.14664 & 0.18445 \\
& $(3)$ & 0.04354 & 0.08121 & 0.13804 & 0.17520 \\
& $(4)$ & 0.04914 & 0.09067 & 0.15072 & 0.18846
\end{tabular}




\begin{tabular}{ccccc}
\hline \hline & $n=50$ & $n=100$ & $n=250$ & $n=500$ \\
\hline$(5)$ & 0.04500 & 0.08747 & 0.14849 & 0.18663 \\
(6) & 0.08530 & 0.13154 & 0.19150 & 0.22693 \\
\hline
\end{tabular}

(c) $x=50$

\begin{tabular}{|c|c|c|c|c|c|}
\hline & & $n=50$ & $n=100$ & $n=250$ & $n=500$ \\
\hline \multirow[t]{6}{*}{$y=10$} & (1) & 0.04546 & 0.07871 & 0.12641 & 0.15631 \\
\hline & (2) & 0.03953 & 0.07195 & 0.11967 & 0.14995 \\
\hline & (3) & 0.03677 & 0.06704 & 0.11263 & 0.14238 \\
\hline & (4) & 0.04149 & 0.07486 & 0.12304 & 0.15326 \\
\hline & (5) & 0.03790 & 0.07204 & 0.12099 & 0.15153 \\
\hline & (6) & 0.07263 & 0.10975 & 0.15786 & 0.18624 \\
\hline \multirow[t]{6}{*}{$y=25$} & (1) & 0.06985 & 0.12068 & 0.19358 & 0.23925 \\
\hline & (2) & 0.06072 & 0.11029 & 0.18322 & 0.22946 \\
\hline & (3) & 0.05648 & 0.10276 & 0.17242 & 0.21787 \\
\hline & (4) & 0.06374 & 0.11475 & 0.18837 & 0.23454 \\
\hline & (5) & 0.05820 & 0.11038 & 0.18519 & 0.23184 \\
\hline & (6) & 0.11173 & 0.16847 & 0.24200 & 0.28535 \\
\hline \multirow[t]{6}{*}{$y=50$} & (1) & 0.08179 & 0.14106 & 0.22605 & 0.27928 \\
\hline & (2) & 0.07109 & 0.12889 & 0.21391 & 0.26781 \\
\hline & (3) & 0.06612 & 0.12008 & 0.20130 & 0.25427 \\
\hline & (4) & 0.07461 & 0.13411 & 0.21994 & 0.27374 \\
\hline & (5) & 0.06811 & 0.12896 & 0.21618 & 0.27056 \\
\hline & (6) & 0.13092 & 0.19709 & 0.28282 & 0.33335 \\
\hline \multirow[t]{6}{*}{$y=\infty$} & (1) & 0.08797 & 0.15150 & 0.24257 & 0.29961 \\
\hline & (2) & 0.07645 & 0.13841 & 0.22951 & 0.28726 \\
\hline & (3) & 0.07111 & 0.12895 & 0.21598 & 0.27273 \\
\hline & (4) & 0.08024 & 0.14401 & 0.23598 & 0.29364 \\
\hline & (5) & 0.07323 & 0.13846 & 0.23192 & 0.29018 \\
\hline & (6) & 0.14089 & 0.21181 & 0.30368 & 0.35782 \\
\hline
\end{tabular}

(d) $x=\infty$

\begin{tabular}{llcccc}
\hline \hline & & $n=50$ & $n=100$ & $n=250$ & $n=500$ \\
\hline$y=10$ & (1) & 0.05649 & 0.09486 & 0.15125 & 0.18753 \\
& (2) & 0.04979 & 0.08740 & 0.14388 & 0.18063 \\
& (3) & 0.04632 & 0.08148 & 0.13556 & 0.17171 \\
& (4) & 0.05222 & 0.09080 & 0.14777 & 0.18444 \\
& (5) & 0.04862 & 0.08804 & 0.14589 & 0.18295 \\
& (6) & 0.08668 & 0.12882 & 0.18538 & 0.21976 \\
$y=25$ & (1) & 0.08982 & 0.15015 & 0.23936 & 0.29716 \\
& (2) & 0.07936 & 0.13856 & 0.22793 & 0.28649
\end{tabular}




\begin{tabular}{|c|c|c|c|c|c|}
\hline & & $n=50$ & $n=100$ & $n=250$ & $n=500$ \\
\hline & (3) & 0.07382 & 0.12920 & 0.21483 & 0.27244 \\
\hline & (4) & 0.08321 & 0.14391 & 0.23403 & 0.29245 \\
\hline & (5) & 0.07773 & 0.13971 & 0.23122 & 0.29026 \\
\hline & (6) & 0.13688 & 0.20292 & 0.29226 & 0.34701 \\
\hline \multirow[t]{6}{*}{$y=50$} & (1) & 0.10822 & 0.18040 & 0.28782 & 0.35798 \\
\hline & (2) & 0.09583 & 0.16672 & 0.27438 & 0.34545 \\
\hline & (3) & 0.08914 & 0.15549 & 0.25870 & 0.32866 \\
\hline & (4) & 0.10046 & 0.17310 & 0.28162 & 0.35253 \\
\hline & (5) & 0.09412 & 0.16826 & 0.27845 & 0.35010 \\
\hline & (6) & 0.16386 & 0.24260 & 0.35003 & 0.41644 \\
\hline \multirow[t]{6}{*}{$y=\infty$} & (1) & 0.11979 & 0.19950 & 0.31943 & 0.39895 \\
\hline & (2) & 0.10637 & 0.18475 & 0.30500 & 0.38557 \\
\hline & (3) & 0.09895 & 0.17235 & 0.28774 & 0.36712 \\
\hline & (4) & 0.11147 & 0.19171 & 0.31287 & 0.39325 \\
\hline & (5) & 0.10479 & 0.18665 & 0.30965 & 0.39085 \\
\hline & (6) & 0.18001 & 0.26657 & 0.38623 & 0.46143 \\
\hline
\end{tabular}

TABLE 3

COMPARISON OF VALUES OF $\operatorname{Pr}\{\mathcal{T} \leq t \mid \mathcal{u}(0)=v\}$ CORRESPONDING TO THE DiCKSON AND Willmot (2005) ERLANG-2 EXAMPLE

\begin{tabular}{c|ccc|ccc}
\hline \hline & \multicolumn{3}{|c|}{$v=1$} & \multicolumn{3}{c}{$v=10$} \\
\hline$t$ & Exact & Approx. 1 & Approx. 2 & Exact & Approx. 1 & Approx. 2 \\
\hline 2 & 0.3649 & 0.3545 & 0.3662 & 0.0002 & 0.0001 & 0.0001 \\
4 & 0.4830 & 0.4732 & 0.4845 & 0.0014 & 0.0012 & 0.0012 \\
6 & 0.5459 & 0.5378 & 0.5484 & 0.0042 & 0.0037 & 0.0038 \\
8 & 0.5863 & 0.5801 & 0.5900 & 0.0084 & 0.0076 & 0.0078 \\
10 & 0.6150 & 0.6104 & 0.6199 & 0.0137 & 0.0126 & 0.0129 \\
20 & 0.6889 & 0.6899 & 0.6978 & 0.0464 & 0.0450 & 0.0459 \\
40 & 0.7416 & 0.7482 & 0.7548 & 0.1038 & 0.1067 & 0.1080 \\
\hline \hline
\end{tabular}


TABLE 4

Approximated values of $\operatorname{Pr}\{\mathcal{T}<t, \mathcal{U}(\mathcal{T}-) \leq x,|\mathcal{U}(\mathcal{T})| \leq y \mid \mathcal{U}(0)=1\}$ CORRESPONDING to THE Dickson AND Willmot (2005) ERLANG-2 EXAMPLE

(a) $t=2$

\begin{tabular}{l|cccccc}
\hline \hline & $x=0.5$ & $x=1$ & $x=1.5$ & $x=2$ & $x=4$ & $x=\infty$ \\
\hline$y=0.5$ & 0.01965 & 0.06694 & 0.14710 & 0.17517 & 0.18520 & $0.18520(0.18465)$ \\
$y=1$ & 0.03376 & 0.10712 & 0.22706 & 0.26814 & 0.28260 & $0.28260(0.28166)$ \\
$y=2$ & 0.04526 & 0.13726 & 0.28381 & 0.33314 & 0.35030 & $0.35030(0.34904)$ \\
$y=3$ & 0.04775 & 0.14346 & 0.29501 & 0.34582 & 0.36345 & $0.36345(0.36212)$ \\
$y=4$ & 0.04821 & 0.14459 & 0.29700 & 0.34806 & 0.36576 & $0.36576(0.36443)$ \\
$y=5$ & 0.04829 & 0.14478 & 0.29734 & 0.34843 & 0.36615 & $0.36615(0.36481)$ \\
$y=\infty$ & 0.04831 & 0.14482 & 0.29740 & 0.34851 & 0.36622 & $0.36622(0.36489)$ \\
\hline
\end{tabular}

(b) $t=6$

\begin{tabular}{l|cccccc}
\hline \hline & $x=0.5$ & $x=1$ & $x=1.5$ & $x=2$ & $x=4$ & $x=\infty$ \\
\hline$y=0.5$ & 0.03072 & 0.10281 & 0.20677 & 0.25122 & 0.27760 & $0.27789(0.27677)$ \\
$y=1$ & 0.05274 & 0.16472 & 0.32022 & 0.38525 & 0.42317 & $0.42358(0.42175)$ \\
$y=2$ & 0.07068 & 0.21125 & 0.40119 & 0.47926 & 0.52417 & $0.52465(0.52227)$ \\
$y=3$ & 0.07456 & 0.22083 & 0.41723 & 0.49765 & 0.54375 & $0.54425(0.54176)$ \\
$y=4$ & 0.07529 & 0.22257 & 0.42010 & 0.50090 & 0.54721 & $0.54770(0.54519)$ \\
$y=5$ & 0.07541 & 0.22286 & 0.42058 & 0.50145 & 0.54778 & $0.54828(0.54576)$ \\
$y=\infty$ & 0.07544 & 0.22292 & 0.42067 & 0.50155 & 0.54789 & $0.54839(0.54587)$ \\
\hline
\end{tabular}

(c) $t=10$

\begin{tabular}{l|cccccc}
\hline \hline & $x=0.5$ & $x=1$ & $x=1.5$ & $x=2$ & $x=4$ & $x=\infty$ \\
\hline$y=0.5$ & 0.03499 & 0.11651 & 0.22951 & 0.28031 & 0.31379 & $0.31438(0.31204)$ \\
$y=1$ & 0.06007 & 0.18673 & 0.35574 & 0.43003 & 0.47815 & $0.47899(0.47531)$ \\
$y=2$ & 0.08049 & 0.23954 & 0.44595 & 0.53514 & 0.59211 & $0.59308(0.58842)$ \\
$y=3$ & 0.08492 & 0.25041 & 0.46385 & 0.55571 & 0.61419 & $0.61519(0.61033)$ \\
$y=4$ & 0.08574 & 0.25238 & 0.46704 & 0.55936 & 0.61808 & $0.61908(0.61419)$ \\
$y=5$ & 0.08588 & 0.25272 & 0.46758 & 0.55997 & 0.61873 & $0.61973(0.61483)$ \\
$y=\infty$ & 0.08591 & 0.25278 & 0.46768 & 0.56008 & 0.61886 & $0.61985(0.61495)$ \\
\hline \hline
\end{tabular}

